\title{
Caracterización del crecimiento del fruto y producción de tres híbridos de tomate (Solanum lycopersicum L.) en tiempo fisiológico bajo invernadero
}

\section{Characterization of fruit growth and yield in three tomato hybrids (Solanum lycopersicum L.) in physiological time under greenhouse conditions}

GUSTAVO ARDILA R. ${ }^{1}$

GERHARD FISCHER ${ }^{2}$

HELBER ENRIQUE BALAGUERA-LÓPEZ ${ }^{3,4}$

Racimo de tomate larga vida

híbrido Beverli.

Foto: G. Ardila R.

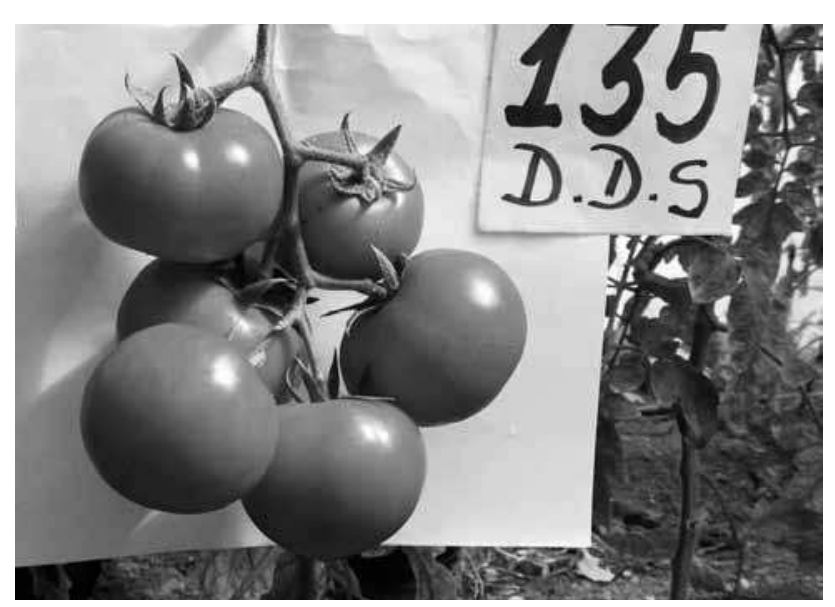

\section{RESUMEN}

Mediante un análisis funcional de crecimiento, es posible establecer el patrón de crecimiento de los frutos, así como determinar la velocidad y dinámica de este proceso. El objetivo fue realizar el estudio del crecimiento del fruto y la producción de tres híbridos de tomate larga vida, Beverly, Franco y SVR, en tiempo fisiológico, bajo cubierta plástica en San Antonio del Tequendama, Cundinamarca (Colombia). Se utilizó un diseño en bloques completos al azar, donde los bloques correspondieron al híbrido de tomate. Cada bloque tuvo cuatro repeticiones; los seis tratamientos correspondieron a la ubicación del fruto en el primer racimo. Las mediciones se realizaron cada 15 días desde la formación del primer fruto hasta la cosecha; la cosecha se realizó hasta el racimo 12. El crecimiento del fruto se ajustó a un modelo logístico. El híbrido Franco presentó los frutos de mayor diámetro, seguido de Beverly. La posición del fruto en el primer racimo mostró un comportamiento diferencial, siendo el fruto 1 el de mayor tamaño y el fruto 6 el más pequeño. El híbrido SVR tuvo un buen comportamiento en la producción y aunque no presentó alta cantidad de extra, sí se caracterizó por una gran producción de calidad primera y segunda, mientras que el Franco presentó los frutos de mayor tamaño pero menor producción que SVR. Beverly tuvo la menor producción y el menor calibre de frutos.

\footnotetext{
Facultad de Agronomía, Programa de Maestría en Ciencias Agrarias con énfasis en Fisiología de Cultivos, Universidad Nacional de Colombia, Bogotá (Colombia).

2 Facultad de Agronomía, Departamento de Agronomía, Universidad Nacional de Colombia, Bogotá (Colombia).

3 Facultad de Agronomía, Doctorado en Ciencias Agropecuarias, Área Agraria con énfasis en Fisiología de Cultivos, Universidad Nacional de Colombia, Bogotá; Facultad de Ciencias Agropecuarias, Grupo de Investigaciones Agrícolas, Universidad Pedagógica y Tecnológica de Colombia, Tunja (Colombia)

4 Autor para correspondencia. ghardilar@hotmail.com
} 
Palabras clave adicionales: híbridos Beverly, Franco y SVR, tasas de crecimiento, rendimiento.

\section{ABSTRACT}

Using a functional analysis, it is possible to establish the pattern of fruit growth and determine its speed and dynamics. The objective of this research was to study the growth of the fruit and the yield of three long shelf-life tomato hybrids, Beverly, Franco and SVR, in physiological time in a greenhouse in San Antonio del Tequendama, Cundinamarca (Colombia). A completely randomized block design was used. The blocks corresponded to the tomato hybrids, each block had four replications; six treatments corresponded to the location of the fruit in the first cluster. Measurements were taken every 15 days from the formation of the first fruit until harvest; the harvest was done after cluster 12. Fruit growth corresponded to a logistic model. The Franco hybrid presented fruits with the largest diameter, followed by Beverly. The position of fruit in the first cluster had a different behavior; with fruit 1 having the largest size and 6 the smallest. The SVR hybrid had a good yield and although it did not produce a large amount of extra, it was characterized by a high yield of first and second quality, while Franco presented the largest size fruits, but a lower yield than SVR. Beverly had the lowest yield and the smallest fruit size.

Additional key words: Beverly, Franco and SVR hybrids, growth rates, production.

El tomate de mesa es la hortaliza con mayor área cultivada debido al mayor consumo a nivel nacional e internacional. En 2008, a nivel mundial se tenían sembradas 5.227 .883 ha con una producción de 129.649.883 t (FAO, 2009), mientras que en Colombia, para el mismo año, el área cultivada fue de 14.855 ha con una producción de 455.693 t (Agronet, 2009). Para los agricultores del país, el cultivo del tomate presenta, entre otras ventajas, una producción en corto tiempo, no se necesita una gran extensión de terreno y se adapta a diferentes tipos de suelos (Barraza et al., 2004).

El tomate es una especie que posee gran diversidad de genotipos, esto indica variantes en los componentes fisiológicos, los cuales determinan en gran parte la eficiencia biológica y la produc- tividad, así como las prácticas de manejo agronómico apropiadas para un mejor desarrollo del cultivo. En el municipio de San Antonio del Tequendama y sus alrededores se están introduciendo nuevos híbridos de tomate larga vida como el Beverly, Franco y SVR, materiales de los cuales se desconoce su comportamiento fisiológico en dicha zona.

El crecimiento se puede referir a un incremento irreversible de materia seca o volumen, cambios en tamaño, masa, forma o número de estructuras, como una función del genotipo y el ambiente (Krug, 1997), dando como resultado un aumento cuantitativo del tamaño y peso de la planta o de un órgano. Es un proceso complejo que incluye muchos fenómenos como división celular, elon- 
gación, fotosíntesis, síntesis de otros compuestos, respiración, translocación, absorción y transpiración (Gómez et al., 1999). El crecimiento puede ser cuantificado mediante el empleo de un conjunto de índices basados en modelos definidos mediante expresiones o funciones matemáticas (Hunt, 1990).

Según Rodríguez (2005), el crecimiento durante un intervalo de tiempo puede ser calculado por simple sustracción. Sin embargo, a partir de las medidas de materia seca en intervalos de tiempo definidos es posible determinar tasas que explican la dinámica del crecimiento en frutos. Las principales evaluaciones son: tasa absoluta de crecimiento (TAC) y tasa relativa de crecimiento (TRC), la TAC indica el cambio de tamaño por unidad de tiempo, mientras que la TRC expresa la tasa de variación del tamaño por unidad de tamaño inicial (Hunt, 1990).

Las curvas de crecimiento del diámetro transversal en función del tiempo son las más empleadas (Casierra y Cardozo, 2009). Tienen la ventaja de ser de fácil determinación, no destructivas y permiten el seguimiento a lo largo del periodo de crecimiento. Estas curvas de crecimiento sirven para identificar no solamente la evolución del crecimiento en el ciclo bajo las condiciones climáticas de la zona de estudio, sino también para estimar el peso que tendrá el fruto en la cosecha (Coombe, 1976; Hunt, 1990).

Entre los órganos vertedero, el fruto está definido como el vertedero prioritario en el contexto de competencia por fotoasimilados (Link, 2000). Dicha habilidad puede estar determinada por varios factores, como el transporte en el floema, metabolismo, compartimentalización (Ho, 1992; 1996) y el tamaño del vertedero, el cual está determinado más por el número de células que por el tamaño de las mismas (Cowan et al., 1997). Además, el tamaño final del fruto está estrechamente relacionado con numerosos parámetros, como el número de cárpelos del ovario, él número de semillas, la posición del fruto en el racimo, la posición del racimo en la planta y de las condiciones ambientales imperantes durante la fase de crecimiento del racimo (Kinet y Peet, 1997).

De acuerdo con López (2009), en la agricultura la combinación del tiempo y la temperatura resulta en el denominado tiempo térmico o también conocido como suma de calor, grados día, grados día de desarrollo, unidades de calor (Ruiz-Corral et al., 2002) o tiempo fisiológico, y se define como la cantidad de grados día necesarios para finalizar un determinado proceso de desarrollo o fase fenológica (Trudgill et al., 2005); se utiliza para el cálculo de la tasa de aparición de nudos, hojas, inflorescencias y desarrollo de frutos (Rodríguez y Flórez, 2006; NeSmith, 1997; Normand y Léchaudel, 2006; Almanza et al., 2010); y para estimar la producción potencial (Salazar et al., 2007), entre otros.

El uso del tiempo fisiológico incrementa la precisión para determinar la duración del ciclo de desarrollo (Ruiz et al., 1998) de una planta o de un órgano, pero solo se logra si el cálculo se hace con valores cercanos a las temperaturas umbrales (máxima y mínima) que controlan el desarrollo de la especie (Ruiz-Corral et al., 2002) pues temperaturas que excedan estos límites van a detener el desarrollo del vegetal (Trudgill et al., 2005). En el caso del tomate la temperatura umbral mínima o temperatura base es de $10^{\circ} \mathrm{C}$ (Rangel y Silvas, 1987; Zotarelli et al., 2009).

El objetivo de este trabajo fue evaluar el crecimiento del fruto y la producción de los híbridos de tomate larga vida Beverly, Franco y SVR 565 en tiempo fisiológico, cultivado bajo cubierta plástica, en condiciones agroecológicas de San Antonio del Tequendama (Cundinamarca).

\section{MATERIALES Y MÉTODOS}

El experimento se realizó en el municipio de San Antonio del Tequendama (Cundinamarca), en 
la vereda Laguna Grande finca La Despensa, situada a $4^{\circ} 38^{\prime} 02,55^{\prime \prime} \mathrm{N}$, y a $74^{\circ} 20^{\prime} 07,57^{\prime \prime} \mathrm{O}$ del meridiano de Greenwich, a $1.900 \mathrm{msnm}$. Se utilizó un lote bajo cubierta plástica (invernadero) de $2.520 \mathrm{~m}^{2}$ donde se sembró un cultivo comercial de tomate larga vida. Dentro del invernadero se presentó una temperatura promedio de $20,2^{\circ} \mathrm{C}$, máxima promedio de $25,64^{\circ} \mathrm{C}$ y mínima promedio de $15^{\circ} \mathrm{C}$.

Se utilizó un diseño en bloques completos al azar con tres bloques, los bloques correspondieron al híbrido de tomate larga vida (Beverly, Franco y SVR 565), cada bloque tuvo cuatro repeticiones, compuesta $\mathrm{c} / \mathrm{u}$ por tres plantas de tomate, los tratamientos correspondientes a la ubicación del fruto en el primer racimo (fruto 1 , 2, 3, 4, 5 ó 6), por tanto, se dejaron seis frutos en el primer racimo, el criterio de selección de los tratamientos fue tomar como fruto 1 el que cuajó primero y así sucesivamente hasta el fruto 6. Los demás racimos también se dejaron con seis frutos/racimo, las mediciones de crecimiento se realizaron cada 15 días desde la formación del primer fruto hasta que alcanzaron la madurez de consumo (fruto 100\% color rojo).

La densidad de plantación fue de 40.000 plantas ha ${ }^{-1}$, el plan de fertilización se basó en el análisis de suelos y se aplicó mediante fertirrigación, los controles fitosanitarios y demás labores culturales se hicieron de acuerdo con las recomendaciones para la zona.

Para el tiempo fisiológico, se hizo el cálculo de la acumulación de grados día calor (GDC) desde el momento del trasplante de las plantas de tomate en el invernadero, mediante la formula utilizada por Rodríguez y Flórez (2006):

GDC $=\left(\frac{(T \text { máx }+T \text { mín })}{2}\right)-$ Tbase

Donde Tmáx es la temperatura máxima diaria del aire; Tmín la temperatura mínima diaria del aire; la Tbase es la temperatura en que el pro- ceso metabólico del tomate es mínimo. Esta temperatura fue de $10^{\circ} \mathrm{C}$, tal como lo recomiendan Rangel y Silvas (1987) y Zotarelli et al. (2009). La comparación entre GDC y días después del trasplante (ddt) se observa en la tabla 1.

\section{Tabla 1. Equivalencia entre días después del trasplante (DDT) y acumulación de grados día calor (GDC).}

\begin{tabular}{|r|r|}
\hline DDT & GDC \\
\hline 60 & 632,5 \\
\hline 75 & 797,5 \\
\hline 90 & 948,5 \\
\hline 105 & 1100,5 \\
\hline 120 & 1247,5 \\
\hline 135 & 1398,5 \\
\hline
\end{tabular}

Las variables de crecimiento medidas en el estudio fueron: (a) diámetro del fruto ( $\mathrm{mm}$ ), medición realizada en la zona ecuatorial del fruto a través de un calibrador Vernier, (b) tasa absoluta de crecimiento (TAC) y (c) tasa relativa de crecimiento (TRC) del diámetro del fruto aplicando las ecuaciones de Hunt (1990) (tabla 2). El rendimiento $\left(\mathrm{kg} \mathrm{ha}^{-1}\right)$ se determinó a través de la producción de frutos por cada una de las categorías comerciales (extra, primera, segunda, tercera, cuarta) de acuerdo con el diámetro del fruto (tabla 3), para esto se midieron y pesaron los frutos recolectados de 12 racimos dos veces por semana y se extrapoló.

Se determinó el modelo de crecimiento no lineal tipo logístico siguiendo la metodología descrita por Carranza et al. (2009) empleando el enfoque funcional para dicho modelo. Con los datos obtenidos se realizó un análisis de varianza y se utilizó la prueba de Tukey con una confiabilidad del 95\%. Se graficó el comportamiento de cada una de las variables respecto al tiempo fisiológico, el programa estadístico utilizado fue SAS v. 8.1e (Cary, N.C). 
Tabla 2. Descripción de los parámetros de crecimiento calculados. Adaptado de Carranza et al. (2009).

\begin{tabular}{|l|l|l|l|}
\hline Índice & \multicolumn{1}{|c|}{ Descripción } & \multicolumn{1}{|c|}{ fórmula } & \multicolumn{1}{c|}{ Unidades } \\
\hline TAC & Tasa absoluta de crecimiento & $\mathrm{dD} / \mathrm{dt}$ & $\mathrm{cm} / \mathrm{GDC}$ \\
\hline TRC & Tasa relativa de crecimiento & $(1 / \mathrm{D})(\mathrm{dD} / \mathrm{dt})$ & $\mathrm{cm} \mathrm{cm}^{-1}$ por GDC \\
\hline
\end{tabular}

$\mathrm{D}=$ diámetro ecuatorial $(\mathrm{cm}) ; \mathrm{dD} / \mathrm{dt}=$ variación del diámetro en función del tiempo.

\begin{tabular}{|l|c|}
\hline Tabla 3. & \multicolumn{2}{c|}{$\begin{array}{c}\text { Clasificación de los frutos de tomate de } \\
\text { acuerdo con el calibre. }\end{array}$} \\
\hline \multicolumn{1}{|c|}{ Clasificación } & Diámetro del fruto (mm) \\
\hline Calibre 1 (extra) & -82 \\
\hline Calibre 2 (primera) & 57 a 82 \\
\hline Calibre 3 (segunda) 67 \\
\hline Calibre 4 (tercera & 47 a 57 \\
\hline Calibre 5 (cuarta) & 40 a 47 \\
\hline
\end{tabular}

Adaptado de Escobar y Lee (2001).

\section{RESULTADOS Y DISCUSIÓN}

\section{Diámetro del fruto}

Se encontró que el aumento del diámetro se ajustó a una curva logística (figuras $1 \mathrm{~A}$ y B; tabla 4). Según Monselise et al. (1978), el crecimiento de los frutos de tomate tiende a seguir una curva sigmoidal simétrica. Casierra et al. (2007) describieron el crecimiento de frutos de tomate de los híbridos Sofía, Bravona y Granitio, y en todos encontraron que el incremento del diámetro transversal de los frutos de los tres cultivares describe curvas sigmoidales simples. En frutos de tomate, se han utilizado también otras funciones para modelar el crecimiento, entre estas se destacan la función de Gompertz (Grange y Andrews, 1993), la de Richards (Heuvelink y Marcelis, 1989) y la cúbica (Casierra y Cardozo, 2009; Casierra et al, 2007).

Se presentaron diferencias estadísticas en los seis muestreos, el híbrido Franco se caracterizó por presentar frutos con un diámetro mayor en el primer racimo que los demás híbridos (figura $1 \mathrm{~A}$ ). Al utilizar el diámetro como índice de madurez se podría cosechar el híbrido Franco y SVR desde los $1.100,5$ GDC (105 ddt) y el Beverly a los 1.247,5 GDC (120 ddt), ya que después de este punto el aumento del diámetro no es representativo, esto también indica que el híbrido Beverly es más tardío (figura 1A). En el desarrollo del fruto de tomate hasta alcanzar un $100 \%$ de color rojo se acumularon aproximadamente 766 GDC (75 días).

En la mayoría de los frutos, el desarrollo temprano se divide en tres fases: desarrollo del ovario, división celular y expansión celular (Gillaspy et al., 1993), Casierra y Cardozo (2009) encontraron que estas etapas no se hicieron evidentes en el comportamiento del diámetro de frutos del cv Quindío, debido a que las mediciones se realizaron semanalmente, por lo cual, estos autores recomiendan hacer estas mediciones con mayor frecuencia.

La situación anterior también se observó parcialmente en el crecimiento de los frutos en el presente estudio, para este caso, no se evidencia la fase 1 del crecimiento sigmoidal debido a que los muestreos se realizaron quincenalmente y se inició cuando el fruto tenía un tamaño lo suficientemente grande para medirle el diámetro sin causarle lesiones. Por lo cual, de los 632,5 (60 ddt) a los 1.100,5 GDC (105 ddt) después del trasplante se presentó la fase de rápido crecimiento generada por un aumento en el proceso de elongación celular, desde los 1.100,5 GDC a los 1.398,5 GDC (135 ddt) se llevó a cabo la fase de maduración, pues el incremento del diámetro no fue alto (figuras $1 \mathrm{~A}$ y $1 \mathrm{~B}$ ).

El incremento en los diámetros y en el volumen del fruto de tomate depende de la extensibilidad 

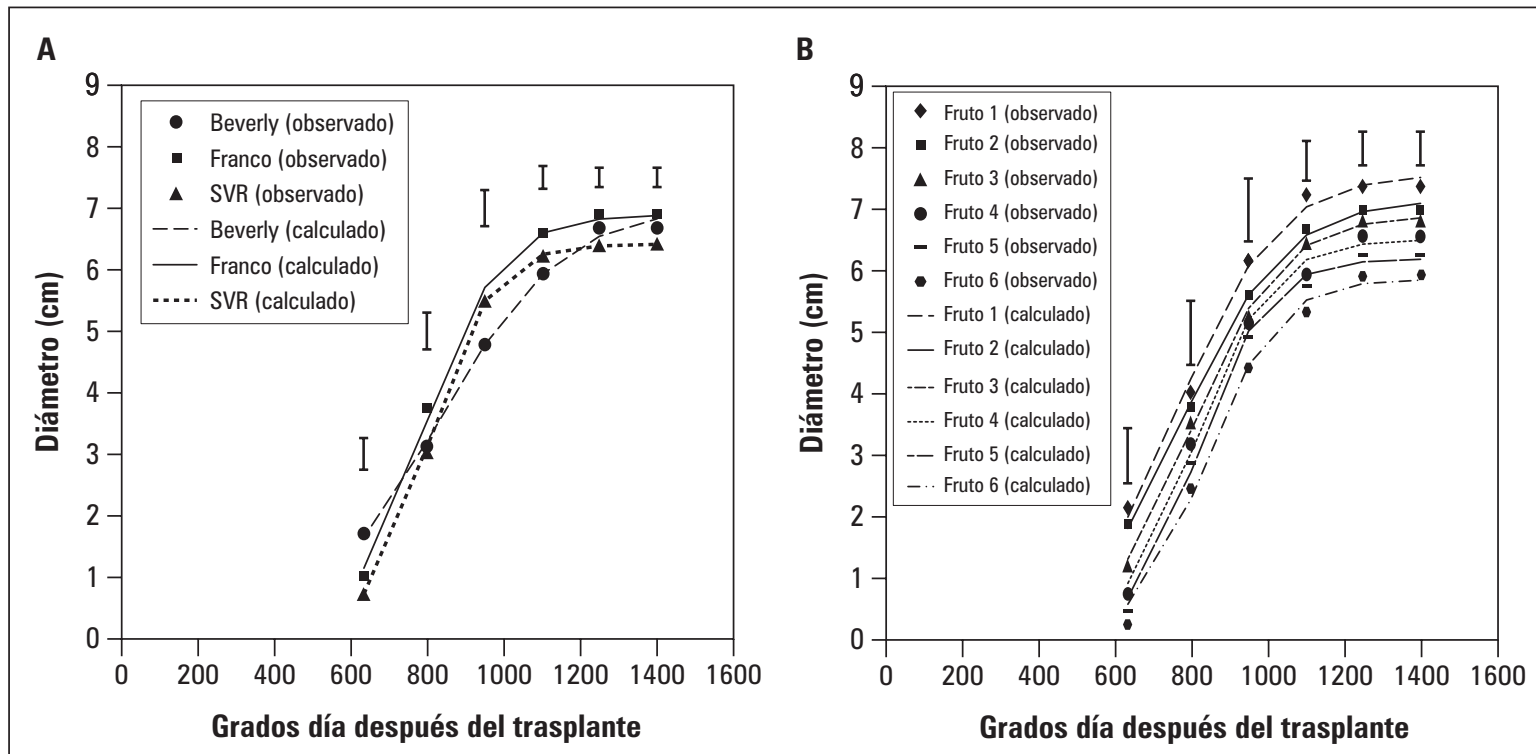

Figura 1. Comportamiento del diámetro del fruto de tomate en tiempo fisiológico de (A) tres híbridos y (B) afectado por la ubicación del fruto en el racimo. La barra representa el valor estadístico (LDS) para comparar los promedios, de acuerdo a la prueba de Tukey. Si las diferencias entre dos promedios son mayores al LDS, entonces habrá diferencia a un $\alpha$ de 0,05 .

Tabla 4. Ecuaciones del modelo logístico para el diámetro durante el crecimiento del fruto de tres híbridos de tomate en tiempo fisiológico.

\begin{tabular}{|c|c|c|}
\hline Tratamiento & Modelo & RSME \\
\hline \multicolumn{3}{|c|}{ Híbridos de tomate larga vida } \\
\hline Híbrido Beverly & $Y=7,027 / 1+e^{-0,00615^{*}(\mathrm{GDC}-826,4)}$ & 0,01730 \\
\hline Híbrido Franco & $Y=6,8901 / 1+e^{-0,0101 *(G D C-792,8)}$ & 0,04800 \\
\hline Híbrido SVR & $Y=6,411 / 1+e^{-0,0122 *(G D C-802,6)}$ & 0,00135 \\
\hline \multicolumn{3}{|c|}{ Ubicación del fruto en el primer racimo } \\
\hline Fruto 1 & $Y=7,5855 / 1+e^{-0,0077^{*}(\mathrm{GDC}-765,9)}$ & 0,04640 \\
\hline Fruto 2 & $Y=7,1743 / 1+e^{-0,00749 *(\operatorname{GDC}-776,5)}$ & 0,01070 \\
\hline Fruto 3 & $Y=6,9096 / 1+e^{-0,0861 *(\operatorname{GDC}-800,8)}$ & 0,02090 \\
\hline Fruto 4 & $Y=6,5199 / 1+e^{-0,0101 *(\operatorname{GDC}-811,1)}$ & 0,04050 \\
\hline Fruto 5 & $Y=6,2031 / 1+e^{-0,0112 *(\operatorname{GDC}-818,9)}$ & 0,04550 \\
\hline Fruto 6 & $Y=5,8724 / 1+e^{-0,0107^{*}(\mathrm{GDC}-839,3)}$ & 0,05300 \\
\hline
\end{tabular}

epidermal (Thompson, 2001) y del desarrollo de los tejidos del pericarpio, los cuales, generalmente comprenden más de las $2 / 3$ partes del total del peso del fruto (Ho y Hewitt, 1986). La división y elongación de los tejidos del pericarpio son determinantes para el crecimiento del fruto de tomate. Mientras que las células epidérmicas se dividen durante todo el desarrollo del fruto, la división en el pericarpio está limitada a un corto periodo del desarrollo del fruto y está lo- 
calizada en los tejidos externos alrededor de los haces vasculares y en la hipodermis, una vez la división celular finaliza, la expansión celular comienza para incrementar el tamaño del fruto (Bertin, 2005). En tomate, las células grandes endorreduplicadas están localizadas en el mesocarpo (Bunger-Kibler y Bangerth, 1983). La endorreduplicacion es un ciclo celular incompleto que permite incrementar el contenido de ADN nuclear (Galbraith et al., 1991) y posiblemente el crecimiento del fruto de tomate (Bunger-Kibler y Bangerth, 1983; Bertin et al., 2003).

Se ha determinado que durante los primeros estados del crecimiento de los frutos el contenido de clorofila total aumenta, con lo cual el fruto presenta una coloración verde, ocurre un crecimiento acelerado y un predominio de la síntesis de clorofilas sobre la de antocianinas (Hernández-Gil y Bautista, 1977), así mismo, la síntesis de hormonas de crecimiento se encuentra estimulada, lo que provoca una rápida división celular; acorde con el incremento en el contenido de auxinas (Coombe, 1976) y giberelinas.

Respecto al comportamiento del diámetro del fruto dependiendo de la ubicación dentro del racimo, se encontró que todas las curvas de ajuste fueron tipo logística. En todos los momentos de muestreo se presentaron diferencias estadísticas, del mismo modo, se determinó que hubo un crecimiento diferencial y descendente a medida que el fruto se encuentra más distante de la base del racimo, a los 1.398,5 GDC (135 ddt) el diámetro de cada uno de los frutos numerados del 1 al 6 fue 7,$38 ; 7,00 ; 6,82 ; 6,56 ; 6,27$ y $5,92 \mathrm{~cm}$ respectivamente (figura 1B). El efecto de la posición del fruto sobre el tamaño del mismo también fue evaluado por Adams et al. (2001), quienes también encontraron que los frutos proximales son más grandes y maduran más rápido que los dístales en los primeros racimos.

Según Adams et al. (2001), el número de células en frutos de tomate depende del desarrollo de la planta, de la relación fuente-vertedero, y la po- sición del fruto, pero el gradiente en el número de células, y por lo tanto, en el peso potencial de frutos proximales a distales dentro del mismo racimo depende en gran medida el nivel de competencia durante el desarrollo floral. En un nivel de competencia bajo, el número de células apenas difirió entre frutos dentro del mismo racimo, y, por tanto, la fuerza de vertedero inicial del fruto después de la división celular no debería ser diferente. Cuando la competencia aumenta, el número de células se reduce en mayor proporción en frutos distales que proximales, lo que explicaría la menor fuerza de vertedero durante estados más avanzados de desarrollo, y luego las diferencias de tamaño del fruto se espera que aumenten hasta la maduración desde frutos proximales que crecen más rápido y después ejercen mayor competencia contra frutos distales.

Factores como el tamaño del vertedero, la época de iniciación relativa de otros vertederos, la localización y distancia de la fuente son determinantes en la fuerza del vertedero en toda la planta (Bangerth y Ho, 1984), lo cual se evidencia claramente en el presente estudio al observar el crecimiento diferencial y significativo de cada uno de los frutos que comprenden el primer racimo.

Entre los destinos de los fotoasimilados existe cierta jerarquía, dentro de la cual algunos órganos sufren una menor reducción en la disponibilidad de asimilados, siendo los frutos menos sensibles que las flores (Wardlaw, 1990). Los frutos son los responsables de dirigir el flujo de asimilados (Gifford y Evans, 1981) y la fuerza de los mismos como destino está dada por su tamaño, potencialmente determinado por la cantidad de células fijadas en la antesis y por su actividad (Ho, 1996).

\section{Tasa absoluta de crecimiento}

La TAC presentó un comportamiento típico de campana en los tres híbridos de tomate, lo cual coincide con lo encontrado por otros autores para frutos de esta misma especie (Casierra y 
Cardozo, 2009; Casierra et al., 2007). Se evidenció un incremento muy alto en los híbridos SVR y Franco de los 632,5 a los 820 GDC, mientras que en Beverly este aumento fue menor; sin embargo, en este híbrido la posterior disminución fue mucho más lenta que en los dos híbridos anteriores, al correlacionarlo con el aumento del diámetro se confirma que en este híbrido el desarrollo del fruto es más tardío (figura 2A).

La ubicación del fruto en el racimo también generó un efecto diferencial en la TAC, no obstante presentó un comportamiento similar, que se caracterizó por mostrar un aumento representativo de los 632,5 a los 800 GDC aproximadamente en los frutos 3 , 4 y 5 donde alcanzaron su máximo valor; por su parte, los frutos 1 y 2 presentaron este aumento mucho más rápido (770 GDC) y el fruto número 6 lo alcanzó más tarde (830 GDC). Después de llegar al punto más alto, la TAC de todos los frutos sufrió una disminución muy rápida hasta la cosecha (1.398,5 GDC) lo que coincide con la fase de maduración de los frutos, no obstante, en los frutos 1 y 2 la disminución fue menor, indicando que son los frutos que alcanza el mayor tamaño dentro del racimo (figura 2B).

El conocimiento de la tasa absoluta de crecimiento es un indicativo del poder vertedero de los frutos (Hunt, 1990; 2003); de acuerdo con esto, Casierra y Cardozo (2009) comentan que es importante reconocer que las labores agrícolas tendientes a mejorar la acumulación de materia seca en los frutos se deben llevar a cabo hasta la mayor TAC, dado que es el periodo en que el fruto es sensible de influenciarse por actividades como la fertilización foliar, el suministro de agua e incluso por un mayor suministro de luz.

\section{Tasa relativa de crecimiento}

Esta tasa de crecimiento presentó su valor más alto a los 632,5 GDC en los tres híbridos, siendo mucho menor en el híbrido Beverly $\left(0,00473 \mathrm{~cm} \mathrm{~cm}^{-1}\right.$ por GDC), posteriormente se observó un descenso que fue mucho más drástico hasta los 1.100,5 GDC para Franco y SVR, pero en Beverly esta disminución fue más lenta (figura $3 \mathrm{~A}$ ).

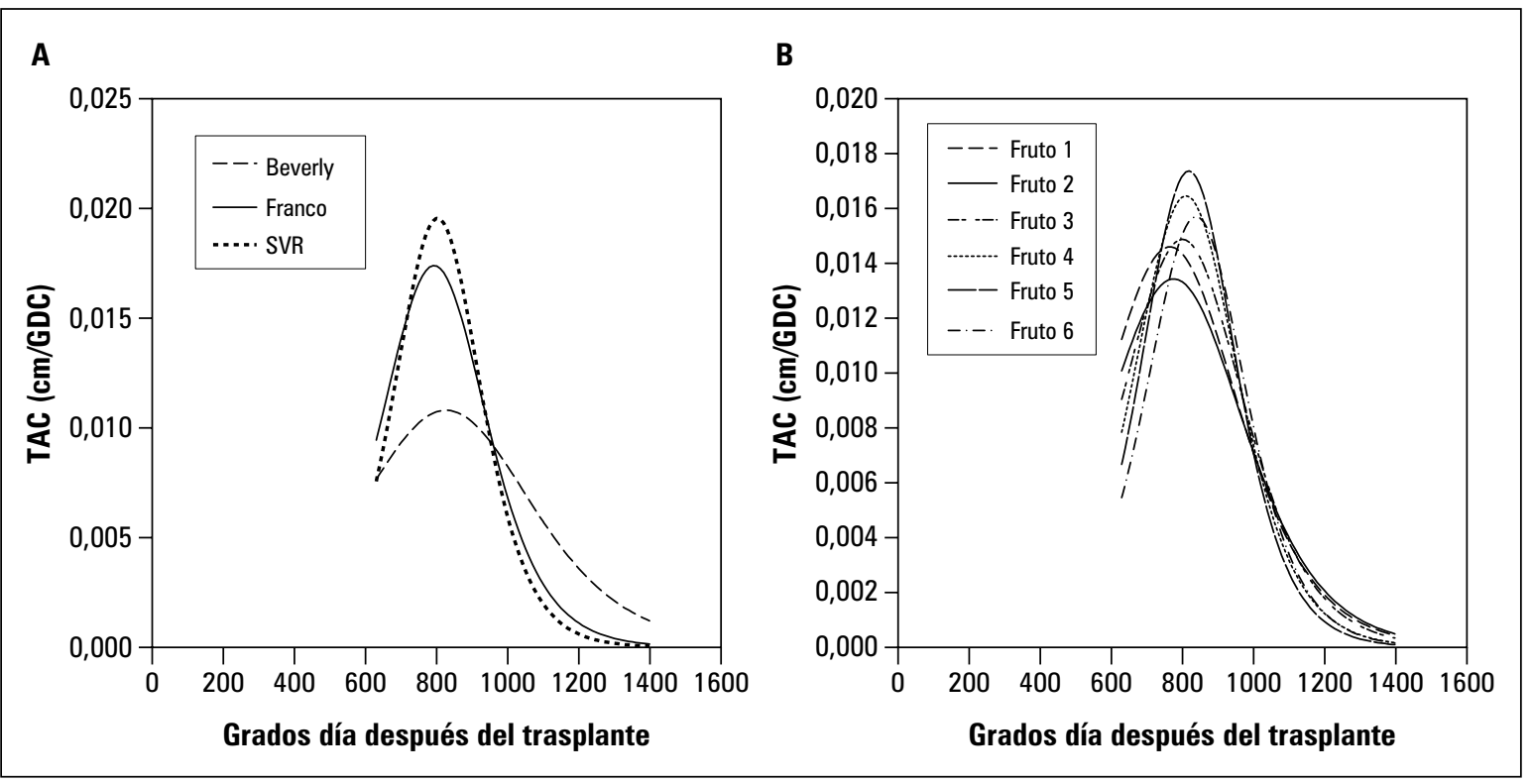

Figura 2. Comportamiento de la tasa absoluta de crecimiento (TAC) del fruto de tomate en tiempo fisiológico de de (A) tres híbridos y (B) afectado por la ubicación del fruto en el primer racimo. 
La TRC afectada por la ubicación de los frutos tuvo un comportamiento similar al generado por el híbrido, a los 632,5 GDC se presentó el mayor valor y fue mucho mayor en los frutos 5 y 6 y menor en los frutos 1 y 2 , sin embargo, la disminución de la TRC en este último fruto fue más lenta en comparación con los demás frutos, los cuales tuvieron una pérdida en el valor de la TRC muy alta, sin embargo, esta disminución fue más lenta a partir de los 1.100,5 GDC (figura 3B).

Por tanto, la mayor eficiencia en el aumento del diámetro del fruto con respecto al material que la produce fue lograda con el híbrido SVR y con el fruto 3. Además, al principio la TRC es mayor porque el material existente para generar nuevo material es menor, pero a medida que la planta crece tiene mayor cantidad de material acumulado y la proporción de nuevo material generado es menor, así el material neto producido sea mayor. Razón que también explica el hecho de que el fruto 1 aunque fue el de mayor tamaño no presentará la mayor TRC, pues el tamaño inicial fue muy grande, lo cual generó que los incrementos relativos no fueran los más altos.
La TRC es un índice de eficiencia que expresa el crecimiento en términos de una tasa de incremento en tamaño por unidad de tamaño y tiempo (Pedroza et al., 1997); en el caso de los frutos estaría indicando la ganancia obtenida como vertedero, pues la mayoría de los fotoasimilados del fruto provienen de las hojas (Casierra y Cardozo, 2009) y una menor parte procede de la fotosíntesis del mismo fruto en su estado verde. De acuerdo con lo anterior, en frutos inmaduros de tomate se ha encontrado que la fotosíntesis no es despreciable (Czarnowski y Starzecki, 1992), y es responsable de cerca de $10 \%$ de la materia seca presente en el fruto (Ho y Grimbly, 1990).

Desde los 632,5 GDC (60 ddt) se inició la fase de rápido crecimiento determinada principalmente por la elongación celular, y es cuando la TRC sufre la disminución más drástica en los tres híbridos y en los diferentes frutos. Desde los 1.100,5 GDC (105 ddt) la planta entró en la fase de maduración, en la cual la ganancia en diámetro no fue representativa, por tanto, la TRC volvió a disminuir lentamente. Un comportamiento similar de la TRC también fue observado por Ca-

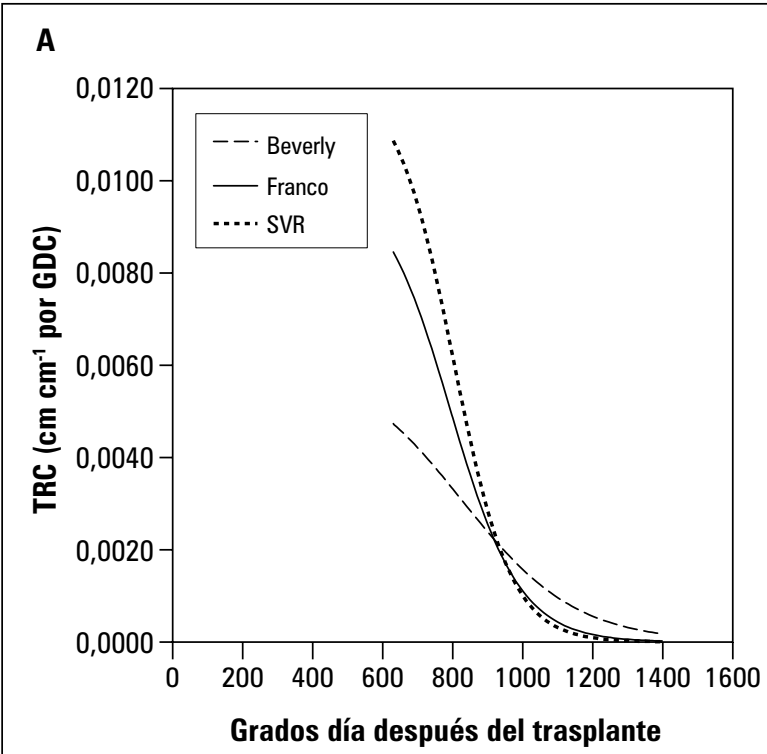

B

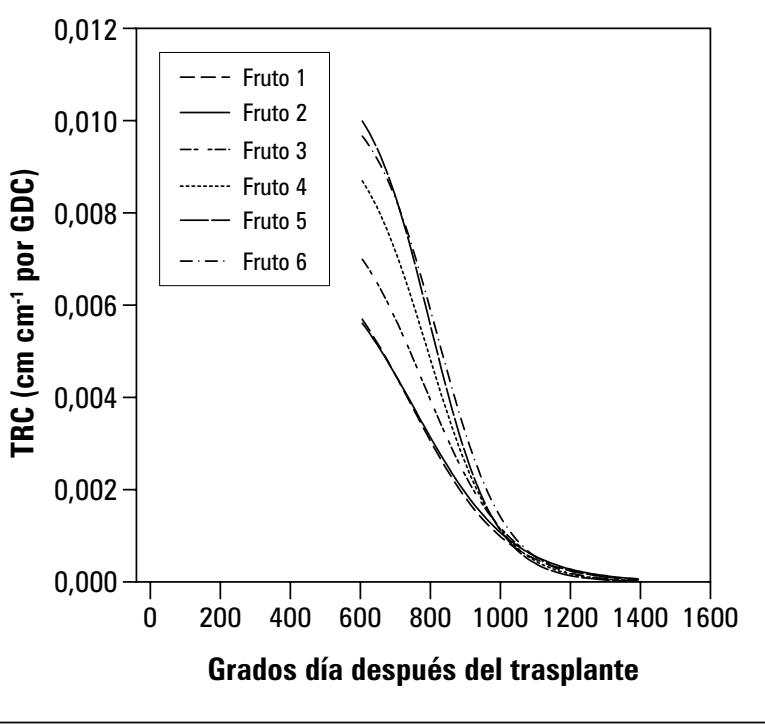

Figura 3. Comportamiento de la tasa relativa de crecimiento (TRC) del fruto de tomate en tiempo fisiológico de (A) tres híbridos y (B) afectado por la ubicación del fruto en el primer racimo. 
sierra y Cardozo (2009) en frutos de tomate cv. Quindío y por Casierra et al. (2007) en frutos de los híbridos de tomate Sofía, Bravona y Granitio, aunque en estos casos las tasas fueron calculadas con base en el peso seco.

\section{Producción}

Se presentaron diferencias estadísticas únicamente en la producción total, a pesar de esto, el híbrido SVR generó mayor producción en las calidades primera $\left(52.667 \mathrm{~kg} \mathrm{ha}^{-1}\right)$ segunda $\left(118.933 \mathrm{~kg} \mathrm{ha}^{-1}\right)$, cuarta $\left(32.933 \mathrm{~kg} \mathrm{ha}^{-1}\right)$ y total (305.533 kg ha-1), mientras que el híbrido franco tuvo mayor producción de calidad extra $\left(8.533 \mathrm{~kg} \mathrm{ha}^{-1}\right)$ y tercera $\left(114.733 \mathrm{~kg} \mathrm{ha}^{-1}\right)$, el híbrido Beverly se caracterizó por generar la menor producción en la mayoría de las calidades (figura 4). No obstante, los tres híbridos presentaron rendimientos que oscilaron entre 257 y 305,5 t ha $^{-1}$, los cuales son altos si se comparan con los reportados por BalagueraLópez et al. (2009) para el híbrido Granitio cultivado en invernadero en el municipio de Santa Sofía (Boyacá), además, el rango para tomate larga vida está entre 110 y 388 t ha ${ }^{-1}$ por ciclo y la producción anual sobrepasa las $500 \mathrm{t} \mathrm{ha}^{-1}$, de tal manera que una planta tiene la capacidad de producir $24 \mathrm{~kg}$ (Ho, 1984).

El rendimiento económico del cultivo del tomate está dado en términos de número de frutos cosechados por unidad de área y sus tamaños individuales (Streck et al., 1998). El tamaño del fruto es un factor de calidad sumamente importante y debe ser lo más uniforme posible durante todo el ciclo de producción, por lo que en términos de producción el mejor híbrido en este estudio fue el SVR, seguido del Franco y por último el Beverly.

Según la ficha técnica de cada uno de los híbridos se puede observar que el rango de peso fresco de los frutos es de 170 a 190 g para Beverly, de 200 a 220 g para Franco y de 160 a 210 g para SVR (Semillas Arroyave, 2010; Compañía Agroindustrial de Semillas, 2010), esto indica junto a los resultados obtenidos, que el Beverly tiene una producción más baja y con frutos de menor tamaño en esta zona, por su parte el SVR tuvo un buen comportamiento en la producción y aunque no tuvo alta cantidad de extra, si se caracterizó por una gran producción de calidad primera y segunda, mientras que el Franco presentó los frutos de mayor tamaño pero menor producción que SVR.

En concordancia, Casierra et al. (2007) afirman que las diferencias encontradas entre los híbridos Sofía, Bravona y Granitio en cuanto a cada una de las variables de crecimiento y calidad de fruto son la consecuencia de la expresión genética de los mismos. Si bien el fenotipo está influenciado por el sistema de producción, el componente genético es determinante en el comportamiento de los cultivares, resultados que concuerdan con los encontrados en este estudio para los híbridos Franco, Beverly y SVR, pues en términos generales, el crecimiento del fruto y la producción fueron diferentes en los tres materiales.

\section{CONCLUSIONES}

El diámetro de los frutos del primer racimo hasta la maduración en los tres cultivares se ajustó a una función logística. El híbrido Franco se caracterizó por presentar frutos con un diámetro mayor seguido del híbrido Beverly, pero este último tuvo un periodo de desarrollo más largo. La posición del fruto en el primer racimo tuvo un comportamiento diferencial e inverso, siendo el fruto 1 el de mayor tamaño y el fruto 6 el más pequeño. El híbrido Beverly presentó menor producción y con frutos de menor tamaño; por su parte el SVR tuvo un buen comportamiento y aunque no tuvo alta cantidad de extra, sí se caracterizó por una gran producción de calidad primera y segunda, mientras que el Franco presentó los frutos de mayor tamaño pero menor producción que SVR. 
A

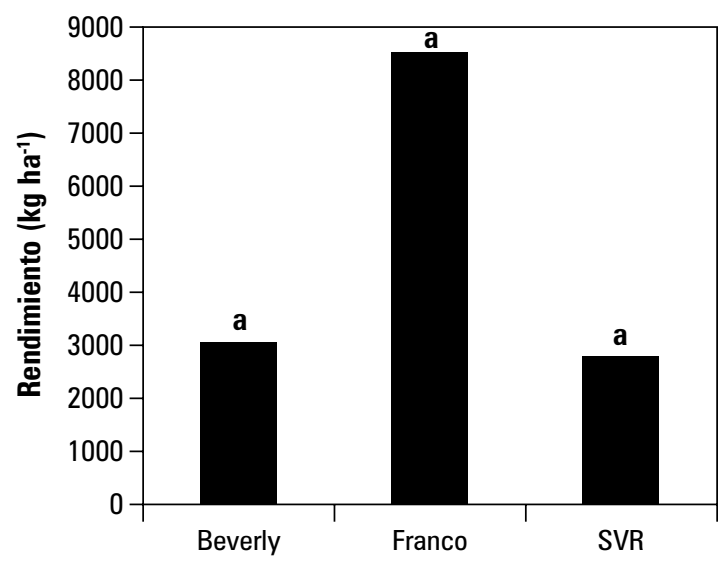

C

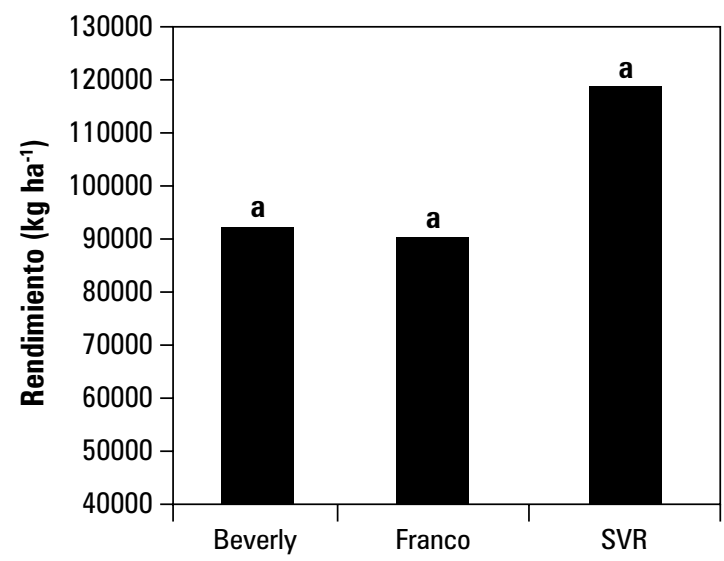

E

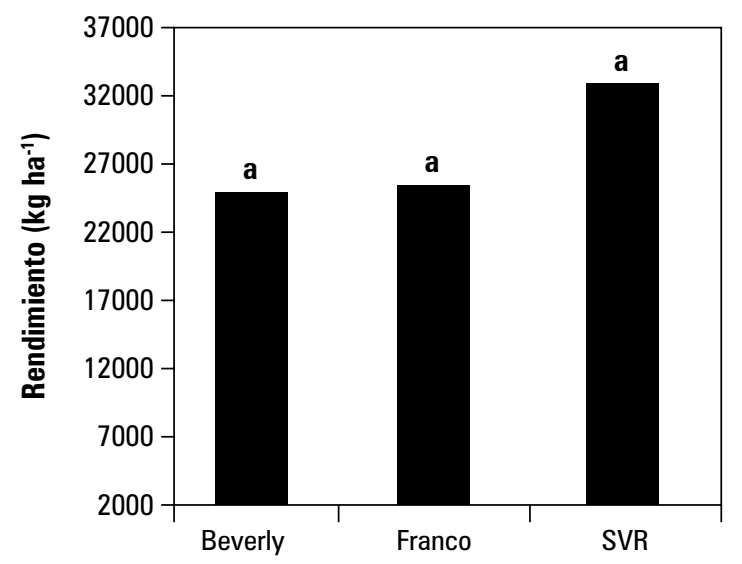

B

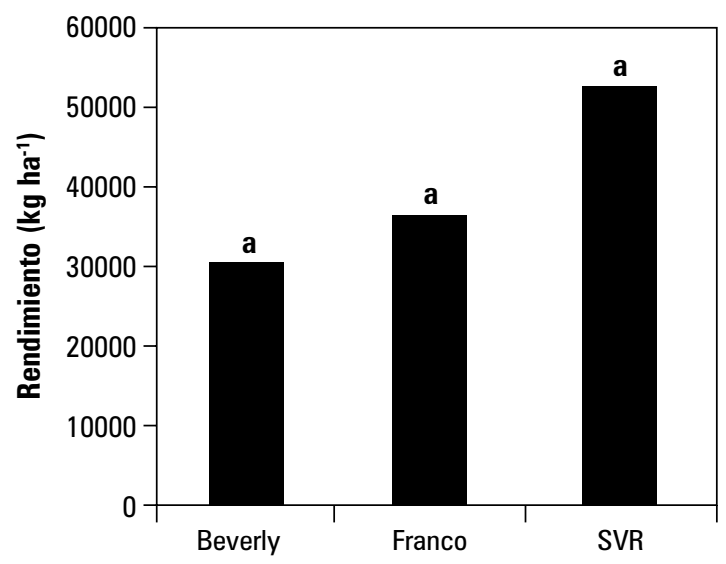

D

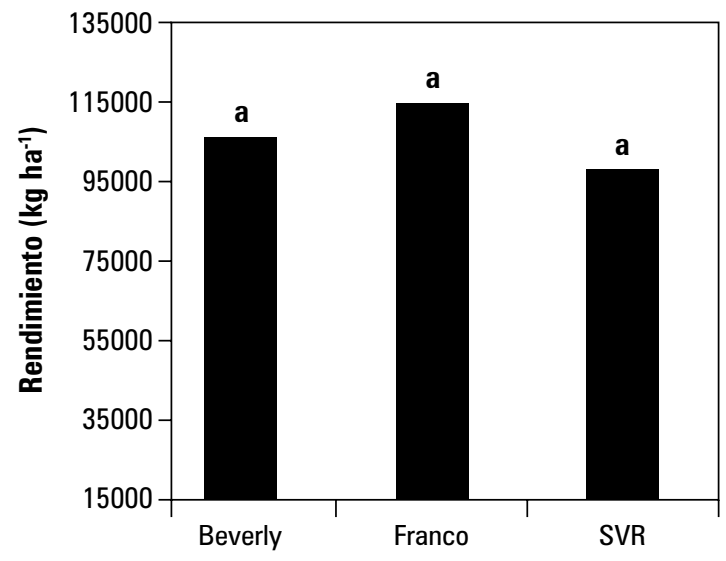

$\mathbf{F}$

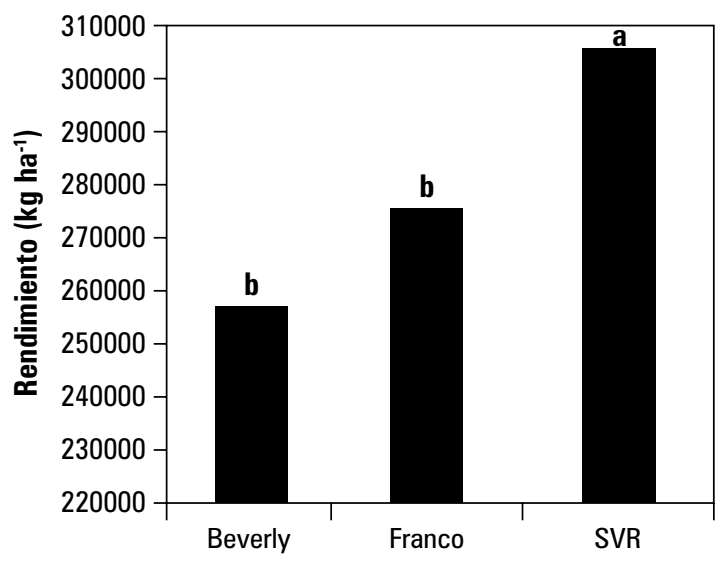

Figura 4. Producción por calidades comerciales de diferentes híbridos de tomate de (A) calidad extra; (B) calidad primera; (C) calidad segunda; (D) calidad tercera; (E) calidad cuarta; (F) producción total. Promedios seguidas de la misma letra no presentan diferencias estadísticas según la prueba de Tukey $(P \leq 0,05)$. 


\section{REFERENCIAS BIBLIOGRÁFICAS}

Adams, S.R., K.E. Cockshull y C.R.J. Cave. 2001. Effect of temperature on the growth and development of tomato fruits. Ann. Bot. 88(5), 869-877.

Agronet. 2009. Área cosechada, producción y rendimiento de tomate En: http://www.agronet.gov.co/; consulta: noviembre de 2009.

Almanza, P., M. Quijano-Rico, G. Fischer, B. Chávez y H.E. Balaguera-López. 2010. Physicochemical characterization during growth and development of grapevine (Vitis vinifera L.) fruits under high tropical conditions. Agron. Colomb. 28(2), 173-180.

Balaguera-López, H.E., J. Álvarez-Herrera, W. Balaguera y G. Martínez. El contenido de arcilla del suelo influye en el rendimiento de un cultivo de tomate (Solanum lycopersicum L.), Rev. Colomb. Cienc. Hort. 3(2), 200-210.

Bangerth, F. y L.C. Ho. 1984. Fruit position and fruit set sequence in a truss as factors determining final size of tomato fruits. Ann. Bot. 53, 315-319.

Barraza, F.V., G. Fischer y C.E. Cardona. 2004. Estudio del proceso del crecimiento del cultivo del tomate (Lycopersicon esculentum Mill.) en el valle del Sinú medio, Colombia. Agron. Colomb. 22(1), 81-90.

Bertin, N., C. Borel, B. Brunel, C. Cheniclet y M. Causse. 2003. Do genetic makeup and growth manipulation affect tomato fruit size by cell number, or cell size and DNA endoreduplication? Ann. Bot. 92, 415-424.

Bunger-Kibler, S. y F. Bangerth. 1983. Relationship between cell number, cell size and fruit size of seeded fruits of tomato (Lycopersicon esculentum Mill.), and those induced parthenocarpically by the application of plant growth regulators. Plant Growth Reg. 1, 143-154.

Carranza, C., O. Lanchero, D. Miranda y B. Chaves. 2009. Análisis del crecimiento de lechuga (Lactuca sativa L.) 'Batavia' cultivada en un suelo salino de la Sabana de Bogotá. Agron. Colomb. 27(1), 41-48.

Casierra, F. y M.C. Cardozo. 2009. Análisis básico del crecimiento en frutos de tomate (Lycopersicon esculentum Mill. cv. Quindío) cultivado a campo abierto. Rev. Fac. Nal. Agr. Medellín 62(1), 4815-4822.

Casierra-Posada, F, M.C. Cardozo y J.F. Cárdenas-Hernández. 2007. Análisis del crecimiento en frutos de tomate (Lycopersicon esculentum Mill.) cultivados bajo invernadero. Agron. Colomb. 25(2), 299-305.
Compañía Agroindustrial de Semillas, 2010. Ficha técnica tomate milano Beverly R Z. En: http://www.agroindustrialdesemillas.com/FrontPageLex/11BrowserPhoto. php?.IdArticulo=182; consulta: julio de 2010.

Coombe, B.G. 1976. The development of fleshy fruits. Annu. Rev. Plant Physiol. 27, 207-228.

Cowan A.K., C.S. Moore-Gordon, I. Bertling y B.N. Wolstenholme. 1997. Metabolic control of avocado fruit growth. Plant Physiol. 114, 511-518.

Czarnowski, M. y W. Starzecki. 1992. Spectral properties and $\mathrm{CO}_{2}$ exchange of tomato fruits. Photosynthetica $27,513-519$.

Escobar. H. y R. Lee. 2001. Producción de tomate bajo invernadero. Cuadernos del Centro de Investigaciones y Asesorías Agroindustriales. Fundación Universidad de Bogotá Jorge Tadeo Lozano, Bogotá. pp. 113117.

FAO. 2010. Faostat. Área cosechada, producción y rendimiento de tomate. En: http://faostat.fao.org/ site/567/DesktopDefault.aspx?PageID $=567 \#$ ancor, consulta: agosto de 2010.

Galbraith, D.W., K.R. Harkins y S. Knapp. 1991. Systemic endopolyploidy in Arabidopsis thaliana. Plant Physiol. 96, 985-989.

Gifford, R.E. y Evans, L.T. 1981. Photosynthesis, carbon partitioning and yield. Annu. Rev. Plant Physiol. 32, 485-509.

Gillaspy, G., H. Ben-David y W. Gruissem. 1993. Fruits: a developmental perspective. Plant Cell 5, 1439-1451.

Gómez, C., C. Buitrago, M. Cante y B. Huertas. 1999. Ecofisiología de papa (Solanum tuberosum) utilizada para cultivo fresco y para la industria. Revista Comalfi 26(1-3), $42-55$.

Grange, R.I. y J. Andrews. 1993. Growth rates of glasshouse tomato in relation to final size. J. Hort. Sci. $68,747-754$

Hernández-Gil, R. y D. Bautista. 1977. Crecimiento y cambios bioquímicos durante el proceso de maduración de la mora (Rubus glaucus Benth.). Agronomía Tropical, 27(2), 225-233.

Heuvelink, E. y L.F.M. Marcelis. 1989. Dry matter distribution in tomato and cucumber. Acta Hort. 260, 149-157. 
Ho, L.C. 1996. The mechanism of assimilate partitioning and carbohydrate compartmentation in fruit in relation to the quality and yield of tomato. J. Exp. Bot. 47, 1239-1243.

Ho. L.C. 1992. The distribution and identity of assimilates in tomato with special reference to stem reserves. Ann. Bot. 73, 315-323.

Ho, L.C. y P. Grimbly. 1990. The physiological basis for tomato quality. Grower 22, 33-36.

Ho, L.C. y J.D. Hewitt. 1986. Fruit development. pp. 201-239. En: Atherton, J.G. y J. Rudich (eds.). The tomato crop. A scientific basis for improvement. Chapman and Hall, London.

Ho, L.C. 1984. Partitioning of assimilates in fruiting tomato plants. Plant Growth Reg. 2, 277-285.

Hunt, R. 2003. Growth analysis, individual plants. pp. 579-588. En: Thomas, B., D.J. Murphy y D. Murray (eds.). Encyclopaedia of applied plant sciences. Academic Press, London.

Hunt, R. 1990. Basic growth analysis. Plant growth analysis for beginners. Unwin Hyman, Boston.

Kinet, J. y M. Peet. 1997. Tomato. pp. 207-258. En: Wien, H.C. (ed.). The physiology of vegetable crops. Cabi Publishing, Wallingford, UK.

Krug, H. 1997. Enviromental influences on development growth and yield. pp. 101-180. En: Wien, H.C. (ed.). The physiology of vegetable crops. Cabi Publishing, London.

Link, H. 2000. Significance of flower and fruit thinning on fruit quality. Plant Growth Reg. 31, 17-26.

López, M.A. 2009. Modelación matemática del crecimiento de clavel estándar cv. Delphi cultivado en sustratos. Tesis de maestría. Facultad de Agronomía, Universidad Nacional de Colombia, Bogotá.

Monselise, S.P., A. Varga y J. Bruinsma. 1978. Growth analysis of the tomato fruit, Lycopersicon esculentum Mill. Ann. Bot. 42, 1245-1247.

NeSmith, S.D. 1997. Summer squash (Cucurbita pepo L.) leaf number as influenced by thermal time. Sci. Hortic. 68, 219-225.

Normand, F. y M. Léchaudel. 2006. Toward a better interpretation and use of thermal time models. Acta Hort. 707, 159-164.

Pedroza, M.J.A., G. Corchuelo y A. Angarita. 1997. Análisis de crecimiento de Limonium sinuatum Mill cv. Midnight Blue propagada sexual y asexualmente a partir de yemas vegetativas y florales. Agron. Colomb. 14(1), 1-12.

Rangel R.J. y J.R. Silvas. 1987. Fenología del tomate en tres fechas de siembra. En: Avances de instigación en hortalizas en el estado de Sinaloa. SARH-INIFAPCAEVACU. Publicación No. 12, 45-47.

Rodríguez, M. 2005. Estudio del crecimiento y desarrollo y de la maduración del fruto de feijoa (Acca sellowiana Berg) en los clones 41 (Quimba) y 8-4, en condiciones del municipio de La Vega (Cundinamarca). Tesis de maestría. Facultad de Agronomía, Universidad Nacional de Colombia, Bogotá.

Rodríguez, W. y V. Flórez. 2006. Comportamiento fenológico de tres variedades de rosas rojas en función de la acumulación de la temperatura. Agron. Colomb. 24(2), 247-257.

Ruiz-Corral, J., H. Flores-López, J. Ramírez-Díaz y D. González-Equiarte. 2002. temperaturas cardinales y duración del ciclo de madurez del híbrido de maíz H-311 en condiciones de temporal. Agrociencia 36, 569-577.

Ruiz C.J.A., J.J. Sánchez y M. M. Goodman. 1998. Base temperature and heat unit requirement of 49 mexican maize races. Maydica 43, 277-282.

Salazar, M.R., J.W. Jones, B. Chaves y A. Cooman. 2007. A model for the potential production and dry matter distribution of cape gooseberry (Physalis peruviana L.) Sci. Hortic. 115, 142-148.

Semillas Arroyave. 2010. Fichas técnicas tomates híbrido Franco y SVR 565. Bogotá.

Streck, N.A., G.A. Buriol, J.L. Andriolo y M.A. Sandri. 1998. Influência da densidade de plantas e da poda apical drástica na produtividade do tomateiro em estufa de plástico. Pesquisa Agropecuária Brasileira 33(7), 1105-1112.

Thompson, D.S. 2001. Extensiometric determination of the rheological properties of the epidermis of growing tomato fruit. J. Exp. Bot.52, 1291-1301.

Trudgill, D.L., A. Honek, D. Li y N.M. Van Straalen, 2005. Thermal time - Concepts and utility. Ann. Appl. Biol. 146, 1-14.

Wardlaw, I. F. 1990. The control of carbón partitioning in plants. New Phytology 116 341-348.

Zotarelli, L., J.M. Scholberg, M.D. Dukes, R. MuñozCarpena y J. Icerman. 2009. Tomato yield, biomass accumulation, root distribution and irrigation water use efficiency on a sandy soil, as affected by nitrogen rate and irrigation scheduling. Agr. Water Manage. 96, 23-34. 